\title{
Brainstem Radionecrosis in a Patient with Nasopharyngeal Carcinoma
}

\author{
Theodora Afrantou, Konstantinos S. Natsis, Aristomenis Angelou
}

Keywords: Oncology, Radiation, Radiation therapy

doi:10.1017/cjn.2017.225

Can J Neurol Sci. 2017; 44: 734-735

A 28-year-old female was admitted with a progressive gait disturbance, difficulty in swallowing, diplopia, and dysphonia. The neurological examination showed severe spastic paraparesis, pyramidal signs on the upper limbs, hoarseness, paresis of the left VI and VII, and bilateral impairment of all the lower cranial nerves. During examination of her medical history, she reported diabetes as well as nasopharyngeal carcinoma, for which she had undergone radiotherapy 1.5 years ago. She received a total dose of 63 Gy in 2-Gy fractions in three phases, which lasted for a period of 7 weeks. MRI revealed an extended lesion to the brainstem from midbrain to the medulla oblongata, which was shown to be hypointense on T1 and hyperintense on T2. This lesion presented a marked homogenous enhancement (Figure 1). The results of her standard biochemical examination were found normal. She was treated with $1 \mathrm{gr}$ of methylprednizolone for 5 days, followed by gradual reduction of the dose. After 2 months of corticosteroid treatment, a mild clinical improvement was observed

Cerebral radionecrosis is one of the late complications of radiation therapy for head and neck tumors. Severe clinical deterioration is characteristic, which in some cases may have a fatal outcome. The exact prevalence of radionecrosis is rather unknown, although an incidence rate of $5-35 \%$ has been reported in patients treated for intracranial tumors. For patients receiving a total dose of $55 \mathrm{~Gy}$, the incidence rate varies between 3 and 5\%. Generally, it is observed 6 months to 2 years after completion of the radiation therapy, and it mainly affects the cerebral white matter. The most commonly affected parts of the brain are the cerebral hemispheres, with a predilection for the temporal lobes and the cerebellum, whereas the brainstem is a rather rare site of involvement. Brainstem lesions include a wide range of differential diagnoses. Based on our patient's imaging, we should take into consideration tumor recurrence, vascular diseases (e.g., stroke, vascular malformations), osmotic demyelination syndrome, infectious rhomboencephalitis associated with Listeria monocytogenes, Bickerstaff brainstem encephalitis, or even primary brainstem gliomas. In the case of our patient, the final diagnosis was based on the clinical and imaging findings and the previous history of radiotherapy, after excluding all other possible diagnoses.

Normal serum sodium levels and negative Listeria antibodies excluded the possibility of osmotic demyelination syndrome and rhomboencephalitis, respectively. Bickerstaff brainstem encephalitis was rather unlikely due to the lack of headache, drowsiness, and flaccid paralysis, which are key features in its clinical presentation. Considering the previous history of radiotherapy, radionecrosis was the most possible scenario. Radiation necrosis can also produce variable and nonspecific clinical symptoms associated with a mass lesion-such as headache, seizures, vertigo, increased intracranial pressure, and focal deficits - always depending on the display area of the necrosis. In nasopharyngeal carcinoma, specifically, the temporal lobes are mainly affected, probably because they are located at the radiation port. Radiation necrosis in the temporal lobes may cause symptoms of memory loss, personality change, or Kluver-Bucy syndrome. Radiotherapy of ocular and maxillary tumors, on the other hand, mainly affect the frontal lobes, resulting in hemiparesis, personality change, or apathy.

So far, more than 300 cases of cerebral injury have been reported after radiation treatment of extracranial malignancies. The most commonly observed primary site is the nasopharynx, followed by the scalp, and the sinonasal tract. ${ }^{1}$ Although the evolution of radiotherapeutic techniques minimizes the amount of normal tissue within the treatment field, it is impossible to totally exclude normal tissue because a minimum margin is required to cover microscopic disease. The severity of the post-radiation injury depends on the total dosing and the fractionation protocol used. ${ }^{2}$ Additionally, such various factors as the number of past surgical procedures, diabetes mellitus, and hypertension are found to be associated with increased risk for brainstem toxicity after radiotherapy. ${ }^{2}$ In general, doses above than $65-70$ Gy and fraction sizes greater than 2 Gy seem to increase the risk for brainstem toxicity. Various therapeutic choices - including resection, steroids, anticoagulants, bevacizumab, and hyperbaric oxygen - have been used to treat cerebral radionecrosis. In our case, the total dose of radiation given was within the therapeutic limits. However, in a number of cases, post-radiation complications were reported even when the total radiation dose was lower than 70 Gy. As already mentioned, our patient presented her neurological symptoms 18 months after completion of radiotherapy. We believe that the coexistence of diabetes mellitus played a major role in the development of the extended brainstem lesion, though this is not the most usual reference among the lesions created after irradiation for extracranial malignancies.

\section{Disclosures}

Theodora Afrantou, Konstantinos Natsis, and Aristomenis Angelou hereby declare that they have no conflicts of interest to disclose.

From the Second Department of Neurology, AHEPA University General Hospital of Thessaloniki, Thessaloniki, Greece.

Received May 31, 2016. Final Revisions Submitted May 31, 2017. Date of Acceptance June 1, 2017.

Correspondence to: Konstantinos Natsis, Second Department of Neurology, AHEPA University General Hospital of Thessaloniki, St. Kyriakidi 1, 54636, Thessaloniki, Greece. Email: kostna1980@gmail.com. 

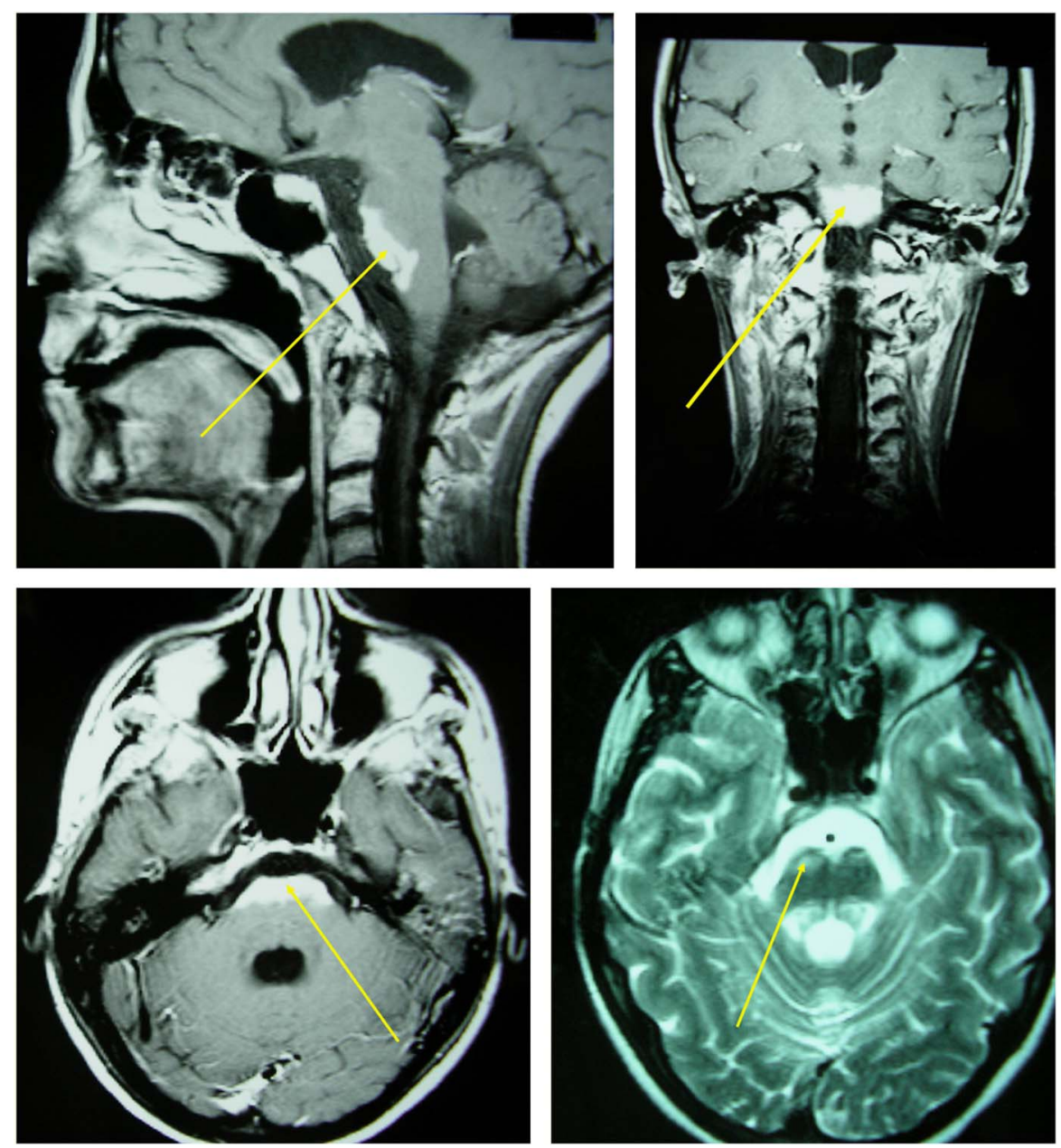

Figure 1: Extended brainstem lesion characterized by $T 2$ hyperintensity and homogenous enhancement in $T 1 C+(G d)$ imaging.

\section{REFERENCES}

1. Hsu YC, Wang LF, Lee KW, Ho KY, Huang CJ, Kuo WR. Cerebral radionecrosis in patients with nasopharyngeal carcinoma. Kaohsiung J Med Sci. 2005;21(10):452-9.
2. Debus J, Hug EB, Liebsch NJ, et al. Brainstem tolerance to conformal radiotherapy of skull base tumors. Int J Radiat Oncol Biol Phys. 1997;39(5):967-75. 